\title{
Ressonância Magnética Funcional em Epilepsia
}

\author{
Maria da Graça Martin* \\ Edson Amaro Jr.** \\ Luiz A. Portela***
}

\begin{abstract}
RESUMO
Atualmente há grande interesse em métodos funcionais não invasivos que poderiam localizar a zona epileptogênica e sua relação com as diversas áreas funcionais corticais. Entre essas novas metodologias, a ressonância magnética funcional é provavelmente um instrumento com potencial para uso na prática clínica. Nós revisamos seus princípios e metodologia.

Unitermos: Ressonância magnética funcional, princípios, metodologia.
\end{abstract}

A ressonância magnética funcional (RMf) tem se tornado método de escolha para diversos estudos de funções cerebrais. Isso se deve, em grande parte, à boa resolução temporal e espacial em comparação a outros métodos baseados no mesmo princípio hemodinâmico, à não necessidade de injeção de materiais de contraste exógenos, possibilidade de repetições do teste e acessibilidade a magnetos de RM capazes de realizar o exame.

Afinal, o que é RMf? É um método que correlaciona a variação do sinal da imagem adquirida por ressonância magnética com uma variação temporal estabelecida, que deve corresponder a uma determinada tarefa desempenhada.

Desse modo, coloca-se um indivíduo no aparelho de ressonância magnética e pede-se que desempenhe determinada tarefa enquanto imagens são adquiridas. Então, estas são submetidas a algum tipo de análise estatística através de um software que irá produzir uma imagem com aquilo que chamamos de "ativações cerebrais".

É importante observar que não é o sinal em si da imagem que determina as áreas de ativação, mas sim a variação do sinal no tempo. Dessa forma, não basta pedir ao indivíduo que movimente os dedos e esperar que apareça a área ativada. É preciso adquirir imagens onde a única diferença seja a movimentação dos dedos. Por exemplo, num estudo de finger tapping em bloco, pode-se ficar 30 segundos movimentando os dedos e 30 segundos com as mãos imóveis. Depois, é necessário um pós-processamento para correlacionar as imagens adquiridas na primeira etapa com as da segunda, e determinar quais áreas tiveram uma variação temporal do sinal na imagem exatamente correspondente à variação temporal da tarefa. Assim, percebe-se que uma parte muito importante do exame está no desenho do paradigma (a série de atividades - tarefas cognitivas simples ou complexas - que o paciente fará durante a aquisição das imagens), pois o ideal consiste em que a única diferença entre a tarefa e o controle seja exatamente aquilo que se quer estudar.

Existem algumas técnicas diferentes de aquisição das imagens para estudos de RMf. A mais usada é aquela baseada no contraste BOLD (blood oxigen level dependent). A técnica BOLD é baseada no uso da desoxi-hemoglobina como contraste paramagnético endógeno.Teoricamente, acredita-se que após um estímulo neural há um aumento do fluxo sangüíneo cerebral local (cerebral blood flow - CBF) maior do que o aumento da taxa de consumo de oxigênio (cerebral metabolic rate for oxygen - $\mathrm{CMRO}_{2}$ ), resultando em

* Neurorradiologista, UNIRAD, Hospital das Clínicas da Faculdade de Medicina da Universidade de São Paulo.

** Neurorradiologista, UNIRAD, Hospital das Clínicas da Faculdade de Medicina da Universidade de São Paulo.

*** Neurorradiologista do Centro de Epilepsia de São Paulo, Hospital Alemão Oswaldo Cruz. 
redução da desoxi-hemoglobina local e conseqüente aumento da homogeneidade do campo magnético local. Tal efeito paramagnético da desoxihemoglobina pode ser medido por técnicas convencionais de imagem pesadas em $\mathrm{T} 2$ e $\mathrm{T} 2 *$.

Não se sabe ainda a exata magnitude da correlação entre o sinal BOLD e a atividade neuronal. Recentemente, Kim ${ }^{1}$ realizou um estudo em que correlacionou o sinal BOLD e a atividade neuronal através de RMf e registro da freqüência de disparos através de um microeletrodo de tungstênio intracelular implantado na mesma área cortical. Neste estudo, ele mostrou que o sinal BOLD no córtex visual primário do gato é bifásico com cada componente do sinal mostrando uma diferente relação funcional com a fisiologia neuronal ${ }^{1}$.

Seus dados sugeriram que há uma relação linear entre as respostas BOLD e neuronal somente se considerado um grande número de áreas de registro. Por outro lado, se forem considerados os registros individuais, as correlações variaram marcadamente, o que permitiu a conclusão de que o sinal BOLD convencional é um indicador fidedigno para atividade neuronal somente para escalas espaciais supramilimétricas. Logothetis et al. ${ }^{2}$ num estudo com registro simultâneo de atividade neural com microeletrodo intracortical e resposta BOLD no córtex visual de macacos, compararam os potenciais locais com a freqüência de disparos e o sinal BOLD e concluíram que o mecanismo de contraste BOLD refletiu diretamente a resposta neural desencadeada por um estímulo. Numa primeira análise, as respostas neural e
BOLD pareceram ter uma relação linear para estímulos de curta duração. Os sinais neurais têm uma relação sinal-ruído muito maior que a resposta hemodinâmica, sugerindo que a extensão da área de ativação em estudos de RMf é geralmente subestimada em relação à atividade neural. Finalmente, a resposta hemodinâmica parece correlacionar melhor com os potenciais locais do que com o somatório de múltiplas unidades. Esses achados sugerem que o mecanismo de contraste BOLD reflete mais o input e o processamento intracortical de uma área determinada do que seu output.

\section{Aplicações clínicas da ressonância magnética funcional em epilepsia}

Desde os primórdios dessa técnica, em estudos experimentais realizados a partir de 1990, a RMf tem se desenvolvido muito e tem contribuído para a melhor compreensão das funções cerebrais. Contudo, o desenvolvimento de aplicações clínicas ainda é restrito.

Entre as limitações desta técnica, figuram: dados insuficientes quanto à natureza do sinal e de seus artefatos; conhecimento insuficiente do funcionamento cognitivo normal para permitir uma aplicação a estados patológicos; desconhecimento de sensibilidade e especificidade para aplicação clínica individual.

Apesar dessas limitações, a sua utilização clínica tem avançado.

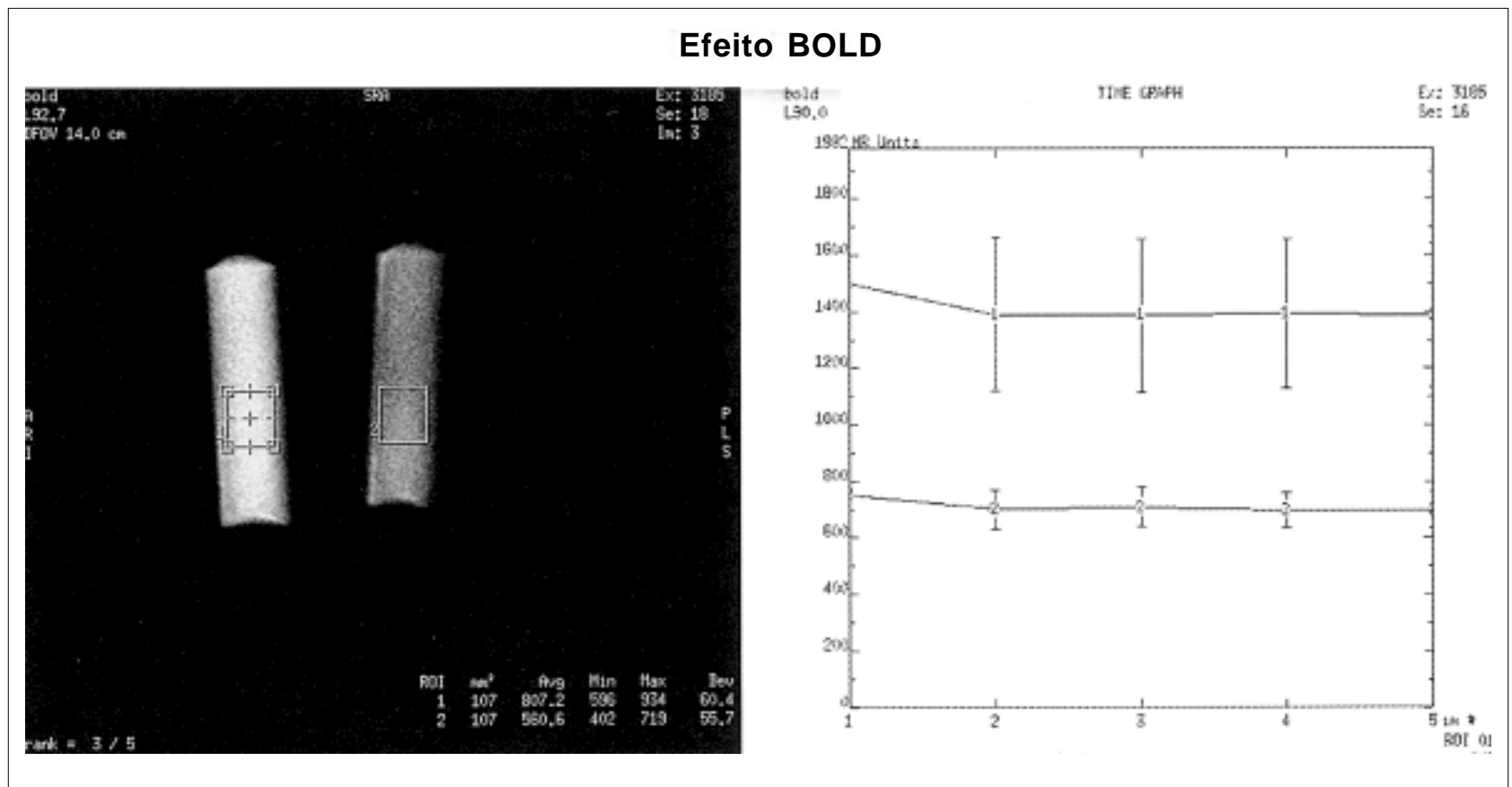

Figura 1 A seringa 1 contém sangue arterial enquanto a seringa 2, sangue venoso. Observe a diferença de sinal em decorrência do aumento na desoxi-hemoglobina. 
Para pacientes com epilepsia, são duas as principais indicações: a determinação de lateralização da linguagem e da memória, principalmente em candidatos a lobectomia temporal e a determinação da zona epileptogênica. Além destas, a técnica pode ser utilizada na determinação da área motora e de linguagem nos casos de lesões focais cirúrgicas. Yetkin et al. ${ }^{3}$ mostraram em um estudo que todas as áreas de ativação mostradas no exame de RMf estavam em torno de $20 \mathrm{~mm}$ do local determinado por eletroestimulação intraoperatória.

\section{Lateralidade de linguagem}

Atualmente, o padrão-ouro para a determinação da lateralidade em linguagem e memória é o teste de Wada ou teste do amobarbital intracarotídeo. Nele, o amobarbital, um barbitúrico de ação ultra-rápida, é injetado através de um cateter na artéria carótida interna, anestesiando temporariamente esse território vascular, usualmente as artérias cerebral média e anterior, as artérias comunicantes anterior e posterior e a artéria coroidea anterior. Durante esse período são realizados testes para determinar as capacidades lingüísticas de cada hemisfério e são apresentadas informações que o paciente deverá recordar ulteriormente, sendo assim testada sua função de memória.

Acredita-se que a RMf seja uma técnica que pode ter um papel importante oferecendo uma alternativa para o teste de Wada, principalmente quanto à linguagem e, talvez, quanto à memória, com a vantagem de ser um método não-invasivo e com a capacidade de determinar não somente o hemisfério dominante, mas também a localização das áreas de interesse (córtex eloqüente) ${ }^{4-10}$.
Sabe-se que as áreas podem variar muito conforme o tipo de estudo utilizado. A distribuição das áreas de linguagem em estudos diferentes não tem sido consistente, o que pode ser atribuído a diversos fatores, como: diferentes tarefas, controles da tarefa, técnica de aquisição, pós-processamento das imagens etc.

Os estudos para determinação de lateralidade na linguagem têm dois paradigmas: geração espontânea de palavras (fluência verbal) e decisão semântica, o primeiro tendendo a ativar consistentemente as áreas anteriores relacionadas à linguagem e o segundo uma rede mais ampla, incluindo áreas anteriores e posteriores.

A tarefa de fluência verbal geralmente é realizada através de um paradigma de geração espontânea de palavras, mas pode ser realizada por geração de palavras de determinada categoria (ex.: animais).

Uma maneira simples e de baixo custo para realizar este tipo de tarefa é projetar estímulos visuais (letras) através da janela de observação da sala de exames numa tela que se encontra próxima aos pés do paciente. Este, por sua vez, poderá visualizar as imagens através de um espelho que se encontra na bobina (a parte do aparelho que fica ao redor da cabeça do paciente) a uma distância de alguns centímetros acima dos seus olhos.

As tarefas de decisão semântica podem ser realizadas tanto por estímulos visuais como auditivos. Por exemplo, pode-se apresentar nomes de objetos versus nomes de animais e solicitar ao paciente que decida se a palavra apresentada representa algo "vivo" ou "morto". É interessante notar que a apresentação de figuras pode confundir o resultado, uma vez que a representação visual de objetos utiliza regiões do hemisfério cerebral direito.

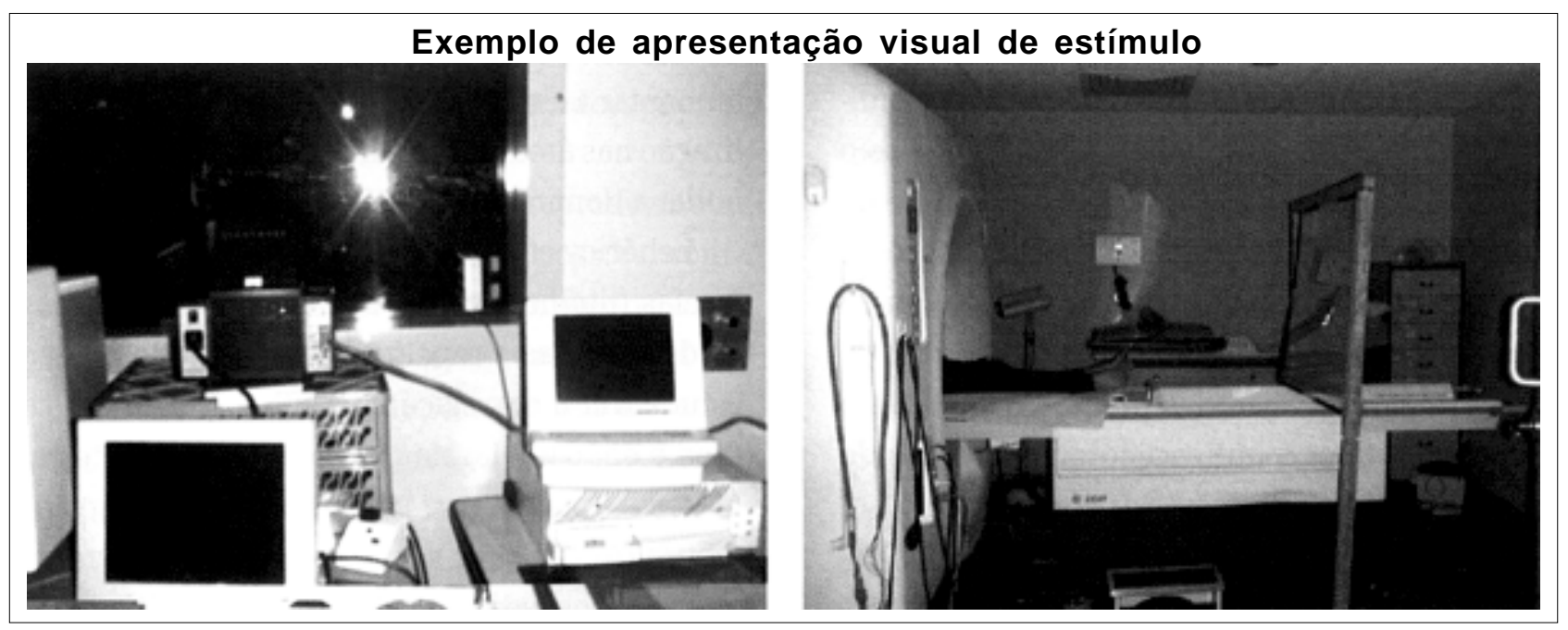

Figura 2 Apresentação visual de palavras através de projetor multimédia em uma tela próxima ao paciente. 
Percebe-se que, devido ao fato dessas tarefas serem realizadas silenciosamente (pois a movimentação da boca gera muitos artefatos), é difícil determinar se o paciente desempenhou a tarefa e, caso o tenha, o foi de forma correta. Para certificar-se de que ele é capaz de executá-la e reduzir o risco de erro, pode-se realizar a tarefa inicialmente fora do magneto, simulando o teste real. Existem formas de estudo com vocalização das palavras, entretanto, esta técnica requer a utilização de adaptações do equipamento, o que pode não ser prático.

Por essas razões, apesar da maior parte dos estudos mostrarem uma ótima correlação entre o teste de Wada e o estudo de fluência verbal ou decisão semântica, é aconselhável a realização de mais de um tipo de paradigma para cada indivíduo.

A tarefa de determinação de rimas tem ótimo valor lateralizatório, e é mais específica para linguagem do que a geração espontânea de palavras por ter um controle mais fino.

Da mesma forma que a fluência verbal, a nomeação de objetos tem um controle menos estável, mas apresenta valor lateralizatório, principalmente nas áreas posteriores.

Também a escolha de áreas de interesse pode aumentar a especificidade do teste, assim, a lateralização nas áreas visuais não é adequada para determinar a dominância hemisférica de linguagem.
Lehericy et al. ${ }^{9}$ num estudo com múltiplas tarefas (fluência verbal semântica, audição passiva de histórias e repetição silenciosa de palavras) estudaram a correlação da lateralização em cada área e em cada tarefa com o resultado do teste de Wada. A audição passiva de histórias mostrou assimetria frontal, mas não temporal, e foi correlacionada com os índices de lateralidade do Wada. A repetição silenciosa de sentenças não teve boa correlação. A fluência verbal semântica mostrou uma assimetria frontal concordante com o Wada - mais acentuadamente nas áreas pré-central, giro frontal médio e sulco frontal inferior.

Num estudo recente, Ramsey et al. ${ }^{11}$ mostraram ótimo resultado utilizando o que eles denominaram análise combinada de tarefas, utilizando um exercício de decisão semântica, geração de antônimo e verbo.

Estes tipos de estudos com múltiplas tarefas parecem mais adequados para a avaliação global da linguagem em cada indivíduo, devendo ser utilizados na prática clínica.

\section{Lateralidade da memória}

A determinação de qual hemisfério é dominante em memória é fundamental para a realização de lobectomia temporal. Os estudos realizados até o momento não mostraram resultados satisfatórios para aplicação clínica

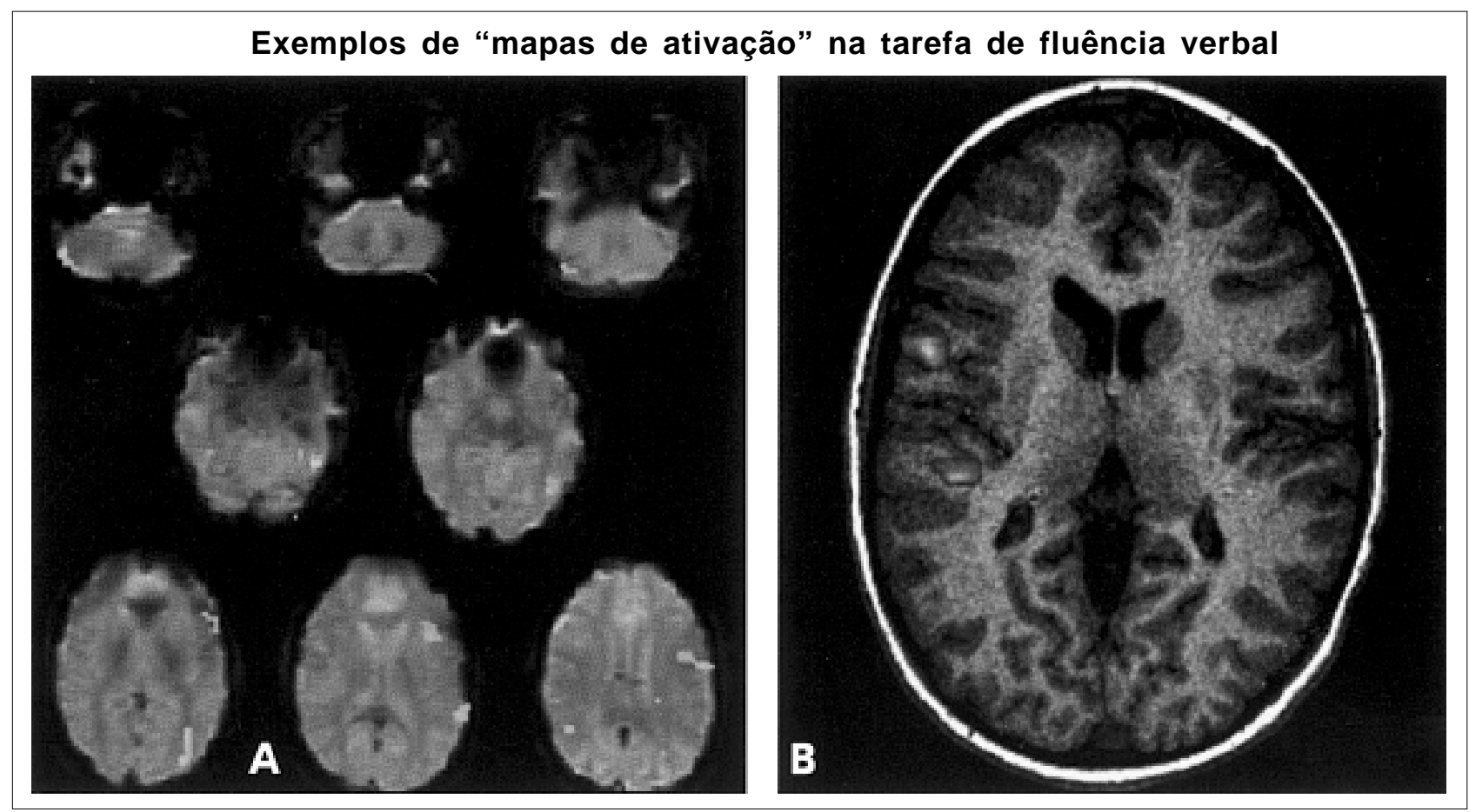

Figura 3 Imagens de ativação em um teste de fluência verbal. A mostra padrão habitual em um teste de geração espontânea de palavras em voluntário destro. B mostra imagem de ativação em um paciente com displasia frontal à esquerda, que realizando a mesma tarefa revela áreas de linguagem à direita sobrepostas em uma imagem de cérebro padrão. O achado foi confirmado no teste de Wada e pela evolução pós-operatória, na qual não foram verificados déficits de linguagem. 


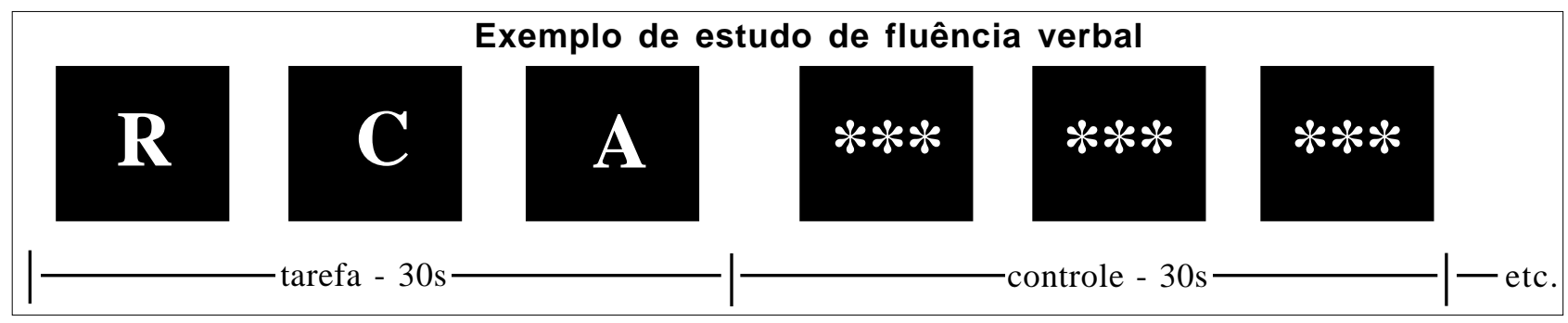

Figura 4 Exemplo simplificado de estudo de fluência verbal. Durante a tarefa são apresentados estímulos visuais com letras a partir das quais a pessoa deve imaginar palavras. Estes são seguidos por um período de descanso no qual se fornece um estímulo neutro que não gere palavras; neste exemplo, asteriscos. Estes períodos são variáveis em cada estudo, com duração de cerca de 30 segundos, que são divididos em dez estímulos diferentes (letras) com três segundos de exposição para cada uma.

individual, e não há uma tarefa totalmente adequada para testes fidedignos de memória.

Além disso, o teste de Wada tenta reproduzir uma condição pós-cirúrgica, o que não é possível com a RMf. Durante o teste de Wada, o hemisfério é anestesiado, o que não pode ser feito com a RMf. Nesta, tenta-se detectar quais circuitos são ativados, mas não se pode prever quais deles são essenciais. Ou seja, a função da RMf é identificar áreas que tenham tido variações hemodinâmicas de acordo com o estímulo, e não quais são as áreas essenciais naquela determinada tarefa; é necessário que não haja nenhuma interposição processual da tarefa com o controle, o que impediria a determinada área de aparecer ativada.

Alguns estudos preliminares foram realizados, como o de Bellgowan et al. ${ }^{12}$, que estudaram 28 pacientes com a tarefa de decisão semântica, encontrando uma assimetria de ativação do lobo temporal mesial muito maior nos pacientes com esclerose mesial temporal direita do que naqueles com esclerose temporal mesial esquerda. Porém, entende-se que no contexto cirúrgico, é importante determinar qual o risco de déficit, o que não foi demonstrado neste estudo.

\section{Identificação da zona epileptogênica}

Os estudos de RMf podem ter como objetivo a identificação da zona epileptogênica. Por essa razão, houve um grande investimento tecnológico no desenvolvimento de equipamentos de EEG compatíveis com RM.

$O$ estudo combinado de RMf com registro eletrencefalográfico torna possível a avaliação de prováveis alterações vasculares detectadas através da seqüência BOLD concomitantemente ao registro EEG para correlação com a zona epileptogênica. $O$ paciente é monitorizado e no início da atividade ictal são adquiridas as imagens. Para a tarefa controle são adquiridas imagens no período interictal.

Em um estudo recente, Lemieux et al. ${ }^{13}$ conseguiram, pela primeira vez, a aquisição contínua de EEG com boa qualidade durante todo o tempo de aquisição de imagens de RMf, possibilitando por meio de melhor resolução temporal e espacial o estudo da zona epileptogênica e da sua propagação. No entanto, além da dificuldade técnica para conseguir todo o equipamento especial, é necessário que o paciente tenha elevada freqüência de descargas e crises relativamente pouco sintomáticas, pois artefatos de movimento impossibilitariam a análise estatística do exame.

\section{SUMMARY}

\section{Functional magnetic resonance in epilepsy}

Presently there is a great interest in new functional non-invasive methods which would be useful in localization of the epileptogenic zone and its relationship with functional cortex. Among these new methods, Functional Resonance Imaging is probably the one with potential to be utilized in clinical practice. We review its principles and methodology.

\section{Keyw ords}

Functional resonance imaging, principles, methodology.

\section{Referências}

1. Kim DS. Points of contact between neuronal physiology and fMRI. Syllabus of " 11 th Advanced Course of the ESNR", Ancona 2001: 53-60. Moonen, B. Functional MRI. Springer, 2000.

2. Logothetis NK, Pauls J, Augath M, Trinath T, Oeltermann A. Neurophysiological investigation of the basis of the fMRI signal. Nature, 412(12):150-7, 2001.

3. Yetkin FZ, Mueller WM, Morris GL et al. Functional MR activation correlated with intraoperative cortical mapping. AJNR, 18:1311-5, 1997.

4. Bahn MM, Lin W, Silbergeld DL et al. Localization of language cortices by functional MR imaging compared with intracarotid amobarbital hemispheric sedation. AJR, 169:575-9, 1997.

5. Benson RR, Fitzgerald DB, LeSueur LL et al. Language dominance determined by whole brain functional MRI 
in patients with brain lesions. Neurology, 52:798$809,1999$.

6. Binder JR, Swanson SJ, Hammeke TA, Morris GL, Mueller WM, Fischer M, Benbadis S, Frost JA, Rao SM, Haughton VM. Determination of language dominance using functional MRI: a comparison with the Wada test. Neurology, 46(4):978-84,1996.

7. Desmond JE, Sum JM, Wagner AD, Demb JB, Shear PK, Glover GH, Gabrieli JD, Morrell MJ. Functional MRI measurement of language lateralization in Wada-tested patients. Brain, 118(6):1411-9, 1995.

8. Bazin B, Cohen L, Lehericy S, Pierrot-Deseilligny C, Marsault C, Baulac M, Le Bihan D. Study of hemispheric lateralization of language regions by functional MRI.Validation with the Wada test. Rev Neurol (Paris), 156(2):145-8, 2000.

9. Lehericy S, Cohen L, Bazin B, Samson S, Giacomini E, Rougetet R, Hertz-Pannier L, Le Bihan D, Marsault C, Baulac M. Functional MR evaluation of temporal and frontal language dominance compared with the Wada test Neurology 54(8):1625-33, 2000.

10. Killgore WD, Glosser G, Casasanto DJ, French JA, Alsop DC, Detre JA. Functional MRI and the Wada test provide complementary information for predicting postoperative seizure control. Seizure, 8(8):450-5, 1999.
11. Ramsey NF, Sommer IEC, Rutten GJ, Kahn RS. Combined analysis of language tasks in fMRI improves assessment of hemispheric dominance for language functions in individual subjects. Neurolmage, 13:71933, 2001.

12. Bellgowan PSF, Binder JR, Swanson SJ, Hammeke TA, Springer JA, Frost JA, Mueller WM, Morris GL. Lateralization of seizure focus predicts activation of the left medial temporal lobe during semantic information encoding: an f MRI study. Neurology 51:479-84, 1998.

13. Lemieux L, Salek-Haddadi A, Josephs O, Allen P, Toms N, Scott C, Krakow K, Turner R, Fish DR. Event-related fMRI with simultaneous and continuous EEG: description of the method and initial case report. Neuroimage, 14:7807, 2001.

\section{Endereço para correspondência:} Hospital Alemão Oswaldo Cruz Rua 13 de Maio, 1815 CEP 01323-001 - São Paulo, SP 